\title{
ON APPROXIMATION BY RATIONALS FROM A HYPERPLANE
}

\author{
GERHARD GIERZ AND BORIS SHEKHTMAN
}

\begin{abstract}
Let $E \subset C(K)$ be a subspace of continuous functions defined on a compact Hausdorff space $K$. We characterize those subspaces of codimension 1 for which the rational functions with denominators and enumerators from $E$ are dense. The condition for the density of this very nonlinear set of functions turns out to be a linear separation condition.
\end{abstract}

A number of recent discoveries in approximation theory indicated some almost supernatural powers of rational approximation (see [1]). That is, starting with a linear subspace $H \subset C([0,1])$, the set of rational functions with the numerator and the denominator in $H$ seems to have density properties disproportionate to its share.

In this note we present the necessary and sufficient condition for the density of such rationals when $H$ is a hyperplane in a $C(X)$-space. Surprisingly enough the condition for the density of this nonlinear set of functions turns out to be a (very linear in spirit) separation condition.

We will need some notation: Let $X$ denote a compact Hausdorff space; $C(X)$ the set of all continuous real-valued functions on $X ; \mathcal{M}(X)$ the space of all regular Borel measures on $X$. The elements from $\mathcal{M}(X)$ are identified with the continuous functionals on $C(X)$. As usual, we define the support supp $f$ of a continuous function $f \in C(X)$ to be the closure of the set $\{x \in X: f(x) \neq 0\}$; the support of a measure $\mu$ on $X$ is defined to be the smallest closed set $A \subset X$ such that supp $f \subset X \backslash A$ implies $\mu(f)=0$. If $U$ is a subset of $X$, we let

$$
\mathcal{F}(U)=\{f \in C(X): \operatorname{supp} f \subset U, f \geq 0\} .
$$

For every $\mu \in \mathcal{M}(X)$ we use $\mu=\mu^{+}-\mu^{-}$to denote the orthogonal decomposition of $\mu$ (i.e., $\mu^{+} \wedge \mu^{-}=0$ ). Recall that for every pair of measures $\mu, \nu \in \mathcal{M}(X)$ their infimum is given by

$$
(\mu \wedge \nu)(f)=\inf \{\mu(g)+\nu(h): 0 \leq g, h, g+h=f\},
$$

where $f \in C(X)$ is positive (see [2, p. 72]). Let $H=\{f \in C(X): \mu(f)=0\}$ for some $\mu \in \mathcal{M}(X)$. Let

$$
R(H)=\left\{g h^{-1}: g, h \in H ; h(x)>0 \forall x \in X\right\} .
$$

LEMMA. Let $\mu \in \mathcal{M}(X)$ and let $U$ be an open subset of $X$ such that $\operatorname{supp} \mu^{+} \cap$ $U \neq \varnothing \neq \operatorname{supp} \mu^{-} \cap U$. Then we can find functions $\varphi_{1}, \varphi_{2} \in \mathcal{F}(U)$ such that $\mu\left(\varphi_{1}\right)<0<\mu\left(\varphi_{2}\right)$.

PROOF. Suppose $\varphi_{1}$ does not exist. Then for all $g \in \mathcal{F}(U)$ we would have $\mu(g) \geq 0$; i.e., $\mu^{+}(g) \geq \mu^{-}(g)$. If $f \in \mathcal{F}(U)$, and if $f=g+h$ for positive functions

Received by the editors January 14, 1985 and, in revised form, April 15, 1985.

1980 Mathematics Subject Classification. Primary 41A20. 
$g$ and $h$, then $g, h \in \mathcal{F}(U)$. Thus $\mu^{+}(g)+\mu^{-}(h) \geq \mu^{-}(g+h)=\mu^{-}(f)$. Hence the above explicit expression for $\mu^{+} \wedge \mu^{-}$yields $0=\left(\mu^{+} \wedge \mu^{-}\right)(f) \geq \mu^{-}(f) \geq 0$ for all $f \in \mathcal{F}(U)$. This would imply $\mu^{-}(f)=0$ whenever supp $f \subset U$; i.e., supp $\mu^{-} \subset$ $X \backslash U$, contradicting $\varnothing \neq U \cap \operatorname{supp} \mu^{-}$.

The proof of the existence of $\varphi_{2}$ is similar.

TheOREM 1. Let $1 \in H$. For the set $R(H)$ to be dense in $C(X)$, it is both necessary and sufficient that

$$
\operatorname{supp} \mu^{+} \cap \operatorname{supp} \mu^{-} \neq \varnothing \text {. }
$$

ProOF. To prove necessity we first assume that $\operatorname{supp} \mu^{+} \wedge \operatorname{supp} \mu^{-}=\varnothing$. Let $f \in C(X)$ be such that $\left.f\right|_{\operatorname{supp} \mu^{+}}=1,\left.f\right|_{\operatorname{supp} \mu^{-}}-1$. If $\|f-g / h\|<1$, then $g$ should be positive on supp $\mu^{+}$and negative on supp $\mu^{-}$(since $h$ is strictly positive). Then $\mu^{+}(g)>0$ and $\mu^{-}(g)<0$, since $\mu^{+}$and $\mu^{-}$are positive measures, and hence $g \notin H$.

To prove sufficiency, let $x_{0} \in \operatorname{supp} \mu^{+} \cap \operatorname{supp} \mu^{-}$, and let $f$ be an arbitrary function from $C(X)$. For $\varepsilon>0$ let $U$ be a neighborhood of $x_{0}$ such that $\mid\left(f\left(x_{0}\right)-\right.$ $f(x)) \mid<\varepsilon / 2$ for all $x \in U$. By the Lemma, there are functions $\varphi_{1}, \varphi_{2} \in \mathcal{F}(U)$ such that $a=\mu\left(\varphi_{1}\right)>0, b=\mu\left(\varphi_{2}\right)<0$. We claim that there exists a function $\varphi \in \mathcal{F}(U) \cap H$ with $\varphi\left(x_{0}\right)>0$. Indeed, pick any $\gamma \in \mathcal{F}(U)$ with $\gamma\left(x_{0}\right)=1$ and let $r=\mu(\gamma)$. If $r<0$, then let $\varphi=a(a-r)^{-1} \cdot \gamma-r(a-r)^{-1} \cdot \varphi_{1}$; if $r>0$, let $\varphi=r(r-b)^{-1} \cdot \varphi_{2}-b(r-b)^{-1} \cdot \gamma$. In any case it follows that $\mu(\varphi)=0$ and $\varphi \in \mathcal{F}(U)$.

Let $\varphi\left(x_{0}\right)=\rho>0$. Let $W \subset U$ be a neighborhood of $x_{0}$ such that $\varphi_{\mid W} \geq \rho / 2$. Using the same argument as above, we obtain a function $\psi \in \mathcal{F}(W)$ such that $\mu(\psi)=-\mu(f)$; i.e., $f+\psi \in H$. Clearly, for an arbitrary integer $N$ the function

$$
r=\frac{f+\psi+N \cdot f\left(x_{0}\right) \varphi}{1+N \varphi} \in R(H) .
$$

Pick $N \geq(4\|\psi\|-2 \varepsilon) /(\varepsilon \rho)$. We obtain the inequality $\|f-r\| \leq \varepsilon$, since

(1) For $x \notin U$, we have $|f(x)-r(x)|=0$.

(2) $x \in U \backslash W$ implies

$$
|f(x)-r(x)|=\frac{N \varphi(x)}{1+N \varphi(x)}\left|f(x)-f\left(x_{0}\right)\right| \leq \frac{\varepsilon}{2} .
$$

(3) $x \in W$ yields

$$
\begin{aligned}
|f(x)-r(x)| & \leq \frac{N \varphi(x)}{1+N \varphi(x)}\left|f(x)-f\left(x_{0}\right)\right|+\frac{\|\psi\|}{1+N \rho / 2} \\
& \leq \frac{\varepsilon}{2}+\frac{\|\psi\|}{1+N \rho / 2} \leq \varepsilon . \quad \square
\end{aligned}
$$

We now obtain two easy generalizations of Theorem 1 . In the first we drop the assumptions about the constants.

THEOREM 2. Let $H=\{f \in C(X): \mu(f)=0\}$ for some $\mu \in \mathcal{M}(X)$. Then $R(H)$ is dense in $C(X)$ iff condition (1) holds.

PROOF. Condition (1) guarantees the existence of a strictly positive function $\varphi \in H$ : Indeed, let $r=\mu(u)$, where $u$ denotes the constant function with value 1. From the Lemma (with $U=X$ ) we conclude that there is a positive function 
$\varphi_{1}$ with $\mu\left(\varphi_{1}\right)=-r$. The function $\varphi=u+\varphi_{1}$ will be strictly positive, and this function belongs to $H$. Consider the hyperplane $H_{\varphi}=\{f / \varphi: f \in H\}$. Then

$$
H_{\varphi}=\left\{f \in C(X): \mu_{1}(f)=0\right\} \text {, }
$$

where $\mu_{1}=\varphi \cdot \mu$. It is easy to see that $\mu_{1}^{+}=\varphi \mu^{+}, \mu_{1}^{-}=\varphi \cdot \mu^{-}$and $1=\varphi / \varphi \in H_{\varphi}$. Hence supp $\mu_{1}^{+} \cap \operatorname{supp} \mu_{1}^{-}=\operatorname{supp} \mu^{+} \cap \operatorname{supp} \mu^{-} \neq \varnothing$, and by Theorem $1, \overline{R\left(H_{\varphi}\right)}=$ $C(X)$. Therefore for every $\varepsilon>0$ and $f \in C(X)$, there exist $g, h \in H$ such that $\|f-(g / \varphi) /(h / \varphi)\|<\varepsilon$, or, equivalently, $\|f-g / h\|<\varepsilon$.

Another obvious generalization of Theorem 1 is as follows.

THEOREM 3. Let $M$ be a finite subset of $\mathcal{M}(X)$ such that for any distinct $\mu, \nu \in M, \operatorname{supp} \mu \cap \operatorname{supp} \nu=\varnothing$. Let $H=\{f \in C(X): \mu(f)=0 \forall \mu \in M\}$. Then $R(H)$ is dense in $C(X)$ iff, for every $\mu \in M$, condition (1) holds.

We now give some examples for Theorem 2.

EXAMPLES. Consider the subspaces

$$
H_{1}=H_{1}(a, b)=\left\{f \in C([0,1]): \int_{0}^{a} f d x=\int_{b}^{1} f d x\right\},
$$

for some $a, b \in(0,1)$,

$$
H_{2}=H_{2}(\varphi)=\left\{f \in C([0,1]): \int_{0}^{1} f \cdot \varphi d x=0\right\} ;
$$

$\varphi \in C\{([0,1])\}$, where $\{\tau: \varphi(\tau)=0\}$ has no interior points,

$$
H_{3}(j)=\operatorname{span}\{\cos k \theta\}_{k=0, k \neq j}^{\infty} \subset C([0, \pi]) .
$$

From Theorem 2 we have

$$
\begin{array}{ll}
\overline{R\left(H_{1}\right)}=C([0,1]) & \text { iff } a=b, \\
\overline{R\left(H_{2}\right)}=C([0,1]) & \text { iff } \varphi \text { changes sign on }[0,1], \\
\overline{R\left(H_{3}\right)}=C([0, \pi]) & \text { iff } j \neq 0 .
\end{array}
$$

The last example follows from the second example and the fact that $H_{3}(j)$ is dense in the hyperplane

$$
H=\left\{f \in C([0, \pi]): \int_{0}^{\pi} f(\theta) \cos j \theta d \theta=0\right\} .
$$

\section{REFERENCES}

1. D. J. Newman, Approximation with rational functions, CBMS Regional Conf. Ser. in Math., no. 41, Amer. Math. Soc., Providence, R. I., 1979.

2. H. H. Schaefer, Banach lattices and positive operators, Springer-Verlag, Berlin and New York, 1974.

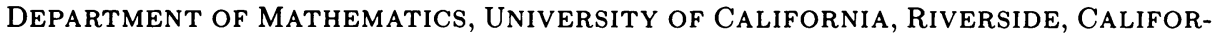
NIA 92521 